\title{
Relationship between markers of inflammation and anaemia in children of Papua New Guinea
}

\author{
Naomi Shinoda', Kevin M Sullivan ${ }^{1,2, *}$ Katie Tripp $^{2}$, Jürgen G Erhardt ${ }^{3}$, \\ Bridgette MH Haynes ${ }^{2}$, Victor J Temple ${ }^{4}$ and Bradley Woodruff ${ }^{2}$ \\ 'Department of Epidemiology, Rollins School of Public Health, Emory University, Claudia Nance Rollins Building \\ (CNR), Room 3051, 1518 Clifton Road NE, Atlanta, GA 30322, USA: ${ }^{2}$ Centers for Disease Control and \\ Prevention, Atlanta, GA, USA: ${ }^{3}$ SEAMEO-TROPMED, University of Indonesia, Jakarta, Indonesia: ${ }^{4}$ School of \\ Medicine and Health Sciences, University of Papua New Guinea, Papua New Guinea
}

Submitted 4 September 2011: Final revision received 2 March 2012: Accepted 15 March 2012: First published online 21 May 2012

\begin{abstract}
Objective: To assess the association of the acute-phase protein biomarkers, C-reactive protein (CRP) and $\alpha_{1}$-acid glycoprotein (AGP), with anaemia in children aged 6-59.9 months in Papua New Guinea.

Design: A nationally representative household-based cross-sectional survey of children aged 6-59.9 months was used to assess the relationships between various combinations of elevated CRP $(>5 \mathrm{mg} / \mathrm{l})$ and AGP $(>1.2 \mathrm{~g} / \mathrm{l})$ with anaemia. Logistic regression was used to determine if other factors, such as age, sex, measures of anthropometry, region, urban/rural residence and household size, modified or confounded the acute-phase protein-anaemia association.

Setting: Papua New Guinea.

Subjects: A total of 870 children aged 6-59.9 months from the 2005 Papua New Guinea National Micronutrient Survey were assessed.

Results: The following prevalence estimates were found: anaemia 48\%; elevated CRP 32\%; and elevated AGP 33\%. Children with elevated CRP had a prevalence of anaemia of $66 \%$ compared with children with normal CRP who had a prevalence of $40 \%$. Corresponding estimates for AGP were $61 \%$ and $42 \%$, respectively. Similar results were found with combinations of elevated CRP and AGP. The higher prevalence of anaemia in children with elevated CRP and/or AGP was still present after controlling for confounders.

Conclusions: Elevated levels of CRP and AGP were significantly associated with a higher prevalence of anaemia in the children surveyed. There are no expert group recommendations on whether to or how to account for markers of inflammation in presenting results on anaemia prevalence. Additional research would be helpful to clarify this issue.
\end{abstract}

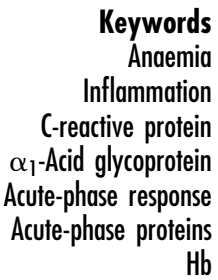

eywords

Anaemia

Inflammation

C-reactive protein Acute-phase response Acute-phase proteins
Anaemia is a major public health problem of global scale. It affects a quarter of the world's population ${ }^{(1)}$ and is common among women and children of pre-school age. Anaemia is an especially significant public health concern in low-income countries. In countries classified by the UN Human Development Index as having a low level of development, anaemia prevalence is $43 \%$, compared with $9 \%$ in those countries classified as having a high level of development ${ }^{(2)}$.

Among the many causes of anaemia are inadequate dietary intake of Fe, increased bodily Fe demand, deficiencies of other vitamins and minerals, and disease status such as inherited haemoglobinopathies and infection ${ }^{(2)}$. In developing countries, malaria, helminth infections and HIV are important determinants of anaemia status ${ }^{(3)}$. Nutritional deficiencies such as Fe deficiency are exacerbated by these diseases, contributing to a cycle of anaemia and infection. This double burden highlights the importance of continued monitoring and assessment of both nutrition and disease status in these areas.

Assessment of micronutrient status can be complicated by the presence of inflammation. Inflammation causes changes in blood concentrations of acute-phase proteins (APP), often occurring concurrently with changes in blood concentrations of micronutrients ${ }^{(4)}$. Thus, the presence of an acute-phase response has the potential to affect the assessment of some nutritional parameters and influence the prevalence estimate of a particular nutrient deficiency. Because infection as well as micronutrient deficiencies tend to be prevalent in many low-income 
countries $^{(3)}$, it is important to determine how the acutephase response of inflammation influences measurements of nutritional status.

C-reactive protein (CRP) and $\alpha_{1}$-acid glycoprotein (AGP) are APP commonly measured to assess the presence of inflammation. CRP concentrations increase quickly in response to an acute insult, peaking at approximately $48 \mathrm{~h}$ and decreasing within a week with a half-life of $19 \mathrm{~h}^{(5)}$. In contrast, AGP concentrations increase more slowly and remain elevated for a longer period of time ${ }^{(6)}$. Taken together, CRP and AGP measurements can be used to classify individuals into four inflammation categories: no inflammation, incubation, early convalescence and late convalescence $^{(7)}$.

While the relationships of APP with serological indicators of vitamin A and Fe status have been studied, few studies have directly addressed the relationship of APP with anaemia. The relationship between current or recent illness and anaemia has been known for years, the so-called 'anaemia of infection' ${ }^{,(8-11)}$. The relationship of biomarkers of inflammation with anaemia prevalence is less studied. Few studies have reported statistically significant relationships between APP and anaemia. In one study, anaemia prevalence was significantly lower in Marshallese children aged 1-5 years without elevated CRP or AGP compared with those with elevated CRP/AGP ${ }^{(12)}$. In a study of HIV-positive postpartum Zimbabwean women, those with elevated AGP had significantly lower $\mathrm{Hb}$ concentrations than those without elevated $\mathrm{AGP}^{(13)}$. Other studies have been less conclusive or have not found associations between APP and anaemia. One study found that children with elevated AGP had a slightly higher prevalence of anaemia in infants $<1$ year of age, although not statistically significant, and no association between elevated CRP and anaemia ${ }^{(14)}$. Another study found no association in pre-school children (aged 1-5 years) between anaemia and a combined marker of inflammation based on TNF- $\alpha$ and $\mathrm{AGP}^{(15)}$.

There are no expert group recommendations on whether to or how to account for APP when presenting the prevalence of anaemia from surveys. The present investigation assessed the association of CRP and AGP with the prevalence of anaemia in Papua New Guinean children aged 6-59.9 months.

\section{Participants and methods}

\section{Study design}

The 2005 Papua New Guinea (PNG) National Micronutrient Survey was a stratified, probability-proportional-to-size cluster survey that was conducted during May-October 2005. The country was divided into four strata (the geographical regions: Southern, Highlands, Momase and Islands) and within each stratum twenty-five clusters were selected using probability proportional to size, with twenty households in each cluster invited to participate in the survey and all eligible individuals within those households invited to participate. Households were randomly selected from communities of 250 or fewer households. For larger communities, the segmentation approach was used to divide the community into segments of approximately equal population size, from which one segment was selected at random and households in that segment randomly selected ${ }^{(16)}$. All children who usually lived in the selected household and were 6-59.9 months of age were considered eligible for the survey. A total of 937 children aged 6-59.9 months were enrolled in the survey, of whom 870 (93\%) had laboratory results for CRP, AGP and $\mathrm{Hb}$.

Verbal consent was obtained and marked on the questionnaires. The survey was approved by a human subjects review board coordinated by the Medical Research and Advisory Committee PNG. The present analysis was based on de-identified data and exempted for review by the Emory University Institutional Review Board.

\section{Blood sampling and testing}

Approximately $250-500 \mu$ l of blood was collected from each participant into a microtainer via a finger stick with a contact-activated sterile lancet with $2.25 \mathrm{~mm}$ needle (Carelet ${ }^{\circledR}$ Safety Lancet; Gainor Medical, Long Beach, CA, USA). Once the blood was collected, the microtainer was gently inverted ten times to prevent clots from forming. $\mathrm{Hb}$ was measured using the HemoCue (HemoCue AB, Angelholm, Sweden). The HemoCue cuvette was filled by holding the microtainer in a horizontal position and carefully tilting the blood forward to the edge of the tube. The pointed tip of the HemoCue cuvette touched the blood drop with the cuvette's groove facing upwards. The cuvette filled automatically by capillary action. Quality control of each HemoCue instrument was performed every morning of the data collection by using control cuvettes. Further, three different levels of liquid controls (high, medium, low) were tested daily in order to ensure correct readings. Anaemia was defined as $\mathrm{Hb}<11 \mathrm{~g} / \mathrm{dl}$ after adjusting for altitude ${ }^{(17)}$. Altitude-adjusted $\mathrm{Hb}$ values $<4 \mathrm{~g} / \mathrm{dl}$ or $>18 \mathrm{~g} / \mathrm{dl}$ were considered extreme and excluded from the analysis ${ }^{(18)}$.

Because of the remoteness of the sampling sites and the difficulty of maintaining a cold chain in the field in PNG, dry blood spots (DBS) were used for the remainder of the blood processing and testing. After use of blood from the microtainer for $\mathrm{Hb}$ and a malaria thick blood smear, DBS were prepared by transferring the remainder of the blood in the microtainers to pre-printed circles on filter papers using a $25 \mu \mathrm{l}$ calibrated micropipette. As many spots as possible were filled on the DBS cards. If blood was left in the microtainer after all spots had been filled, $25 \mu \mathrm{l}$ spots were pipetted in the spaces between the circles, taking care not to allow the spots to overlap. The DBS card was then transferred to a cardboard rack that 
was specially designed for drying DBS filter papers. The box holding the DBS card was left open during the remainder of the stay in the household and a small hand fan was positioned approximately $20 \mathrm{~cm}$ away from the box to help the card dry. The box was closed before being transported to the next house.

Each evening, after the spots were completely dry, the DBS cards were packed in low-gas-permeable bags, with each filter paper separated by glassine (weighing) paper, along with desiccant packs and humidity indicator cards. Each gas-permeable bag was then put in a pleated bag (Tyvek ${ }^{\circledR}$ bags) for storage/shipping. For the duration of storage, humidity indicator cards were monitored every day and desiccant packets were changed as necessary. The DBS cards were sent to Port Moresby where they were packed in dry ice and shipped to the laboratories at the US Centers for Disease Control and Prevention (CDC) in Atlanta, GA for distribution. From CDC they were repackaged and sent to the laboratory at Southeast Asian Ministers of Education Organization - Tropical Medicine and Public Health (SEAMEO-TROPMED) Regional Center for Community Nutrition - University of Indonesia (RCCN-UI) in Jakarta for tests which included CRP and AGP.

Blood concentrations of CRP, AGP and transferrin receptor (TfR) were measured from DBS using an in-house sandwich ELISA ${ }^{(19)}$. The inter-assay variance was $11 \cdot 8 \%$ for CRP, $4.4 \%$ for AGP and $9 \cdot 1 \%$ for TfR. The detection limit for CRP was $0.1 \mathrm{mg} / 1,0.02 \mathrm{~g} / \mathrm{l}$ for AGP and $0.25 \mathrm{mg} / \mathrm{l}$ for TfR. Cut-off thresholds for abnormal values of these proteins vary between studies, and in the case of AGP can vary between 0.8 and $1.4 \mathrm{~g} / \mathrm{l}^{(7)}$. For the present study, inflammation was defined using cut-offs recommended by the laboratory that performed the tests: $5.0 \mathrm{mg} / \mathrm{l}$ for CRP, $1.2 \mathrm{~g} / \mathrm{l}$ for AGP. TfR levels $>8.3 \mathrm{mg} / \mathrm{l}$ were used to indicate Fe deficiency. Combinations of CRP and AGP were assigned according to the conventions of Thurnham et al: no inflammation (normal CRP and AGP), incubation (elevated CRP only), early convalescence (elevated CRP and AGP) and late convalescence (elevated AGP only) ${ }^{(7)}$.

\section{Other variables}

Several variables were assessed to determine if they modify or confound the inflammation-anaemia association. These variables were sex of the child, age group, measures of anthropometry, region (i.e. the stratum), residence in an urban or rural area and household size. Weights and heights were measured in participants and anthropometric indices (stunting, underweight and wasting) were calculated using the WHO 2006 Growth Standards ${ }^{(20)}$. Stunting was defined as height-for-age $Z$-score $(\mathrm{HAZ})<-2$, underweight as weight-for-age $Z$-score (WAZ) $<-2$ and wasting as weight-for-height $Z$-score $($ WHZ $)<-2$. The following extreme values were excluded from analyses: WHZ $<-4$ or $>5$, WAZ $<-5$ or $>5$ and $\mathrm{HAZ}<-5$ or $>3$ as recommended by $\mathrm{WHO}^{(20)}$. Due to technical difficulties, the results of the malaria thick blood smears were determined to be unreliable, and thus not included in the analyses.

\section{Statistical analysis}

The SAS statistical software package version $9 \cdot 1$ for Windows (SAS Institute, Cary, NC, USA) and SAS-Callable SUDAAN Release 9 (RTI International, Research Triangle Park, NC, USA) were used in the statistical analyses. All analyses accounted for the complex survey sample design; sampling weights for each of the four strata were assigned according to population size. Descriptive statistics were obtained to characterize the study population. Logistic regression was used to assess the relationships between anaemia status and each of the following: elevated CRP status, elevated AGP status, presence of any inflammatory response (elevated CRP, elevated AGP or both) and inflammation category. Collinearity diagnostics were conducted by examining condition indices and variance decomposition proportions from the inverse of the information matrix. The SAS macro 'COLLIN' from SAS-L by Matthew Zack was used to calculate collinearity diagnostics from the variance-covariance matrix in non-linear regression models ${ }^{(21)}$. Hierarchical backwards elimination $^{(22)}$ was used to assess for effect modification, confounding and statistically significant independent predictors of anaemia. Interaction terms were created between the measure of APP and other variables. Statistically significant interaction terms and independent predictors were defined by $P<0.05$ and confounding was based on a $10 \%$ rule comparing the 'gold standard' odds ratio from a model controlling for all covariates with the odds ratio from a reduced model $^{(23)}$.

\section{Results}

The number and percentage of participants by various characteristics are shown in Table 1 . Approximately $54 \%$ of the participants were male, $42 \%$ stunted, $18 \%$ underweight and $4 \%$ wasted. The prevalence of Fe deficiency, estimated by TfR, was approximately $27 \%$. Other characteristics not presented in Table 1 include residence in a rural area (81\%) and household size $33 \%$ in households of one to five people; $29 \%$ in households of six or seven people; $38 \%$ in households of eight or more people).

The overall weighted prevalence of anaemia was $48 \%$ (95\% CI 43, 54). Statistically significant predictors of anaemia were elevated CRP, elevated AGP, presence of any inflammation (those with elevated CRP, elevated AGP or both), inflammation category, Fe deficiency, age group, region, wasting and being underweight (Table 1 ). The sex of the child, stunting, urban/rural status and household size were not found to be statistically significant independent predictors of anaemia.

The prevalence of elevated CRP was 32\% (95\% CI 27, 36), elevated AGP 33\% (95\% CI 29, 38) and elevated 
Table 1 Participant characteristics and anaemia prevalence (\%, prevalence odds ratios and $95 \%$ confidence intervals), children 6-59.9 months of age, Papua New Guinea National Micronutrient Survey, 2005

\begin{tabular}{|c|c|c|c|c|c|}
\hline \multirow[b]{2}{*}{ Variable } & \multicolumn{2}{|c|}{ Participant characteristics } & \multicolumn{3}{|c|}{ Anaemia prevalence* } \\
\hline & $n$ & $\%$ & $\%$ & POR & $95 \% \mathrm{Cl}$ \\
\hline \multicolumn{6}{|l|}{ Elevated CRP (>5.0 mg/l) } \\
\hline Yes & 283 & $32 \cdot 5$ & $66 \cdot 3$ & $3 \cdot 0$ & $2 \cdot 1,4 \cdot 2$ \\
\hline No & 587 & $67 \cdot 5$ & $39 \cdot 7$ & $1 \cdot 0$ & Ref. \\
\hline \multicolumn{6}{|l|}{ Elevated AGP $(>1.2 \mathrm{~g} / \mathrm{l})$} \\
\hline Yes & 281 & $32 \cdot 3$ & $61 \cdot 2$ & $2 \cdot 2$ & $1 \cdot 6,3 \cdot 0$ \\
\hline No & 589 & $67 \cdot 7$ & $41 \cdot 7$ & $1 \cdot 0$ & Ref. \\
\hline \multicolumn{6}{|l|}{ Any inflammationt } \\
\hline Yes & 376 & $43 \cdot 2$ & $59 \cdot 9$ & $2 \cdot 3$ & $1 \cdot 7,3 \cdot 1$ \\
\hline No & 494 & $56 \cdot 8$ & $39 \cdot 2$ & $1 \cdot 0$ & Ref. \\
\hline \multicolumn{6}{|l|}{ Inflammation category } \\
\hline No inflammation & 494 & $56 \cdot 8$ & $39 \cdot 2$ & $1 \cdot 0$ & Ref. \\
\hline Incubation & 95 & $10 \cdot 9$ & $55 \cdot 4$ & $1 \cdot 9$ & $1 \cdot 2,3 \cdot 2$ \\
\hline Early convalescence & 188 & $21 \cdot 6$ & $71 \cdot 5$ & 3.9 & $2 \cdot 6,5 \cdot 8$ \\
\hline Late convalescence & 93 & $10 \cdot 7$ & $42 \cdot 1$ & $1 \cdot 1$ & $0 \cdot 7,1 \cdot 8$ \\
\hline \multicolumn{6}{|c|}{ Fe deficiency (DBS TfR $>8.3 \mathrm{mg} / \mathrm{l}) \S$} \\
\hline Yes & 235 & $27 \cdot 1$ & $85 \cdot 3$ & $10 \cdot 6$ & $6 \cdot 8,16 \cdot 3$ \\
\hline No & 632 & $72 \cdot 9$ & $35 \cdot 6$ & $1 \cdot 0$ & Ref. \\
\hline \multicolumn{6}{|l|}{ Sex§ } \\
\hline Male & 465 & $53 \cdot 6$ & $47 \cdot 8$ & $1 \cdot 0$ & $0 \cdot 7,1 \cdot 3$ \\
\hline Female & 402 & $46 \cdot 4$ & $48 \cdot 7$ & $1 \cdot 0$ & Ref. \\
\hline \multicolumn{6}{|l|}{ Age group (months) } \\
\hline $6-11.9$ & 95 & $10 \cdot 9$ & $67 \cdot 4$ & $4 \cdot 2$ & $2 \cdot 2,8 \cdot 0$ \\
\hline $12-23 \cdot 9$ & 208 & $23 \cdot 9$ & $58 \cdot 6$ & $2 \cdot 9$ & $1 \cdot 9,4 \cdot 3$ \\
\hline $24-35 \cdot 9$ & 221 & $25 \cdot 4$ & $43 \cdot 4$ & $1 \cdot 6$ & $1 \cdot 1,2 \cdot 3$ \\
\hline $36-47 \cdot 9$ & 188 & $21 \cdot 6$ & $45 \cdot 4$ & $1 \cdot 7$ & $1 \cdot 1,2 \cdot 6$ \\
\hline $48-59 \cdot 9$ & 158 & $18 \cdot 2$ & $32 \cdot 9$ & $1 \cdot 0$ & Ref. \\
\hline \multicolumn{6}{|l|}{ Region } \\
\hline Southern & 194 & $22 \cdot 3$ & $56 \cdot 7$ & $4 \cdot 0$ & $2 \cdot 0,8 \cdot 0$ \\
\hline Momase & 239 & $27 \cdot 5$ & $67 \cdot 0$ & $6 \cdot 2$ & $2 \cdot 9,13 \cdot 4$ \\
\hline Islands & 242 & $27 \cdot 8$ & $49 \cdot 2$ & $3 \cdot 0$ & $1 \cdot 6,5 \cdot 5$ \\
\hline Highlands & 195 & $22 \cdot 4$ & $24 \cdot 6$ & $1 \cdot 0$ & Ref. \\
\hline \multicolumn{6}{|l|}{ Stunted $(H A Z<-2) \S$} \\
\hline Yes & 350 & $42 \cdot 3$ & $49 \cdot 4$ & $1 \cdot 1$ & $0 \cdot 8,1 \cdot 6$ \\
\hline No & 478 & $57 \cdot 7$ & $47 \cdot 1$ & $1 \cdot 0$ & Ref. \\
\hline \multicolumn{6}{|l|}{ Wasted $(\mathrm{WHZ}<-2) \S$} \\
\hline Yes & 36 & $4 \cdot 3$ & $83 \cdot 9$ & $6 \cdot 0$ & $2 \cdot 5,14 \cdot 4$ \\
\hline No & 806 & $95 \cdot 7$ & $46 \cdot 6$ & $1 \cdot 0$ & Ref. \\
\hline \multicolumn{6}{|l|}{ Underweight $(W A Z<-2) \S$} \\
\hline Yes & 155 & $17 \cdot 9$ & $62 \cdot 2$ & $2 \cdot 1$ & $1 \cdot 4,3 \cdot 1$ \\
\hline No & 711 & $82 \cdot 1$ & $45 \cdot 0$ & $1 \cdot 0$ & Ref. \\
\hline
\end{tabular}

POR, prevalence odds ratio; CRP, C-reactive protein; AGP, $\alpha_{1}$-acid glycoprotein; DBS, dried blood spot; TfR, transferrin receptor; HAZ, height-for-age $Z$ score; WHZ, weight-for-height $Z$ score; WAZ, weight-for-age $Z$ score; Ref., referent category.

${ }^{*}$ Anaemia defined as altitude-adjusted $\mathrm{Hb}<11 \mathrm{~g} / \mathrm{dl}$. Prevalences, POR and $95 \% \mathrm{Cl}$ take into account complex sample design.

tAny inflammation: all participants with elevated CRP, elevated AGP or both.

‡No inflammation, both CRP and AGP normal; incubation, elevated CRP only; early convalescence, both CRP and AGP elevated; late convalescence, elevated AGP only.

\$Due to missing information, sample sizes are as follows: sex, $n 867$; Fe deficiency, $n$ 867; stunting, $n 828$; wasting, $n 842$; underweight, $n 866$.

CRP or AGP 43\% (95\% CI 39, 48). Children who were younger, stunted, underweight, anaemic or Fe-deficient were more likely to have elevated CRP, AGP or both; there were differences by region as well (results not presented). The sex of the child, wasting, urban/rural status and household size were not found to be statistically significant independent predictors of inflammation status.

The prevalence of anaemia was higher in participants with an elevated marker of inflammation compared with those with no indication of inflammation (Table 1). Anaemia prevalence among children with elevated CRP was 66\%, compared with $40 \%$ anaemia prevalence among children without elevated CRP. Similarly, children with elevated AGP had a prevalence of anaemia of $61 \%$ compared with $42 \%$ in children without elevated AGP. Among children with any elevated inflammation markers (elevated CRP, elevated AGP or both), anaemia prevalence was $60 \%$ compared with 39\% in those with no inflammation (both CRP and AGP not elevated). Further, after stratifying the participants into the inflammation category groups as defined by Thurnham et $a l^{(7)}$, those categorized in the incubation and early convalescence inflammation groups had a higher prevalence of anaemia than those with no inflammation.

The relationship between inflammation and anaemia as assessed through logistic regression modelling was still significant after controlling for confounders (Table 2), and 
Table 2 Logistic regression analyses of factors associated with the prevalence of anaemia by infection status, children 6-59.9 months of age, Papua New Guinea National Micronutrient Survey, 2005

\begin{tabular}{|c|c|c|c|c|c|c|c|c|}
\hline \multirow[b]{3}{*}{ Variable } & \multicolumn{8}{|c|}{ Adjusted anaemia prevalence ${ }^{*}$} \\
\hline & \multicolumn{2}{|c|}{$\begin{array}{l}\text { CRP-anaemia } \\
\text { association }\end{array}$} & \multicolumn{2}{|c|}{$\begin{array}{l}\text { AGP-anaemia } \\
\text { association }\end{array}$} & \multicolumn{2}{|c|}{$\begin{array}{l}\text { Any inflammation-anaemia } \\
\text { association }\end{array}$} & \multicolumn{2}{|c|}{$\begin{array}{c}\text { Inflammation } \\
\text { category-anaemia association }\end{array}$} \\
\hline & POR & $95 \% \mathrm{Cl}$ & POR & $95 \% \mathrm{Cl}$ & POR & $95 \% \mathrm{Cl}$ & POR & $95 \% \mathrm{Cl}$ \\
\hline \multicolumn{9}{|l|}{ Elevated CRP (>5 mg/l) } \\
\hline Yes & $2 \cdot 7$ & $1 \cdot 9,4 \cdot 0$ & & & & & & \\
\hline No & $1 \cdot 0$ & Ref. & & & & & & \\
\hline \multicolumn{9}{|l|}{ Elevated AGP (>1.2 mg/l) } \\
\hline Yes & & & $2 \cdot 2$ & $1 \cdot 6,3 \cdot 0$ & & & & \\
\hline No & & & $1 \cdot 0$ & Ref. & & & & \\
\hline \multicolumn{9}{|l|}{ Any inflammationt } \\
\hline Yes & & & & & $2 \cdot 3$ & $1 \cdot 7,3 \cdot 1$ & & \\
\hline No & & & & & $1 \cdot 0$ & Ref. & & \\
\hline \multicolumn{9}{|l|}{ Inflammation category } \\
\hline No inflammation & & & & & & & $1 \cdot 0$ & Ref. \\
\hline Incubation & & & & & & & $1 \cdot 7$ & $1 \cdot 0,3 \cdot 1$ \\
\hline Early convalescence & & & & & & & $3 \cdot 7$ & $2 \cdot 4,5 \cdot 6$ \\
\hline Late convalescence & & & & & & & $1 \cdot 2$ & $0 \cdot 7,2 \cdot 2$ \\
\hline \multicolumn{9}{|l|}{ Age group (months) } \\
\hline $6-11.9$ & $4 \cdot 9$ & $2 \cdot 6,9 \cdot 4$ & & & & & $4 \cdot 7$ & $2 \cdot 5,9 \cdot 0$ \\
\hline $12-23 \cdot 9$ & $3 \cdot 1$ & $2 \cdot 0,4 \cdot 7$ & & & & & $2 \cdot 9$ & $1 \cdot 9,4 \cdot 5$ \\
\hline $24-35 \cdot 9$ & $1 \cdot 4$ & $1 \cdot 0,2 \cdot 1$ & & & & & $1 \cdot 4$ & $0 \cdot 9,2 \cdot 0$ \\
\hline $36-47 \cdot 9$ & $1 \cdot 6$ & $1 \cdot 0,2 \cdot 5$ & & & & & 1.5 & $1 \cdot 0,2 \cdot 4$ \\
\hline $48-59 \cdot 9$ & $1 \cdot 0$ & Ref. & & & & & $1 \cdot 0$ & Ref. \\
\hline \multicolumn{9}{|l|}{ Region } \\
\hline Southern & $4 \cdot 0$ & $2 \cdot 1,7 \cdot 7$ & & & & & $4 \cdot 0$ & $2 \cdot 0,8 \cdot 0$ \\
\hline Momase & $6 \cdot 8$ & $3 \cdot 2,14 \cdot 4$ & & & & & $6 \cdot 2$ & $2 \cdot 9,13 \cdot 4$ \\
\hline Islands & $3 \cdot 1$ & $1 \cdot 7,5 \cdot 6$ & & & & & $3 \cdot 0$ & $1 \cdot 6,5 \cdot 5$ \\
\hline Highlands & $1 \cdot 0$ & Ref. & & & & & $1 \cdot 0$ & Ref. \\
\hline
\end{tabular}

POR, prevalence odds ratio; CRP, C-reactive protein; AGP, $\alpha_{1}$-acid glycoprotein; Ref., referent category

${ }^{*}$ Anaemia defined as altitude-adjusted $\mathrm{Hb}<11 \mathrm{~g} / \mathrm{dl}$. POR and $95 \% \mathrm{Cl}$ take into account weighted analysis.

tAny inflammation: all participants with elevated CRP, elevated AGP or both

¥No inflammation, both CRP and AGP normal; incubation, elevated CRP only; early convalescence, both CRP and AGP elevated, late convalescence, elevated AGP only.

none of the variables investigated acted as effect modifiers of the inflammation-anaemia association. The sex of the child, urban/rural status, household size and the anthropometry indices (stunting, wasting and underweight) were not found to be important confounders in the association between inflammation and anaemia. Age and region remained as confounders in the model assessing the CRP-anaemia association as well as in the model assessing the association between the four inflammation categories and anaemia (Table 2). There were no important confounders for the AGP-anaemia association or for the model assessing the association between any inflammation (participants with elevated CRP, elevated AGP or both) and anaemia (Table 2).

\section{Discussion}

In the present study, children with elevated levels of CRP and AGP, assessed both independently and in combination, were significantly more likely to have anaemia than children without inflammation. There was a strong relationship between the four-level inflammation categorization and anaemia. As presented in Table 2, in the analysis of the association between elevated CRP with anaemia and the four-level inflammation categories with anaemia, age group and region were found to be important confounders. Therefore, at least in these data, failure to account for age group and region can result in overestimating the inflammation-anaemia association. For the AGP-anaemia association and the association between any inflammation (elevated CRP, AGP or both) and anaemia, we did not identify any important confounders.

We did not include the presence/absence of elevated TfR, an indication of $\mathrm{Fe}$ deficiency, in the logistic regression analyses in Table 2 because Fe deficiency could be in the causal pathway between the exposure of interest (inflammation) and the outcome (anaemia). In the assessment of confounding, the potential confounder should not be caused by, or be closely associated with, the exposure of interest ${ }^{(23)}$. In the causal pathway under consideration in the present analysis, inflammation results in elevated CRP and AGP levels. TfR is also affected by inflammation and, as an indicator for Fe deficiency, is also a cause of anaemia. Therefore, TfR is likely on the causal pathway between inflammation and anaemia. The effect of placing an intervening causal variable such as TfR in the logistic regression analyses is a reduction in the inflammation-anaemia association. 
To confirm this, we re-ran the logistic regression analyses for the models shown in Table 2, including TfR as an additional independent predictor. In all four logistic regression models, TfR was a significant predictor of anaemia with adjusted odds ratios in the range of $7 \cdot 1$ to $9 \cdot 6$. Further, even with TfR included in the logistic regression models, there was still a statistically significant relationship between inflammation and anaemia, with the odds ratios for the association between inflammation and anaemia being somewhat reduced. For example, in the model assessing the CRPanaemia association, the adjusted odds ratio for CRP as a predictor of anaemia was $2 \cdot 7$ (controlling for age group and region); with TfR, age group and region in the model, the adjusted odds ratio for CRP was $2 \cdot 3$.

The mechanism by which short-term inflammation as measured by CRP and AGP is associated with lower $\mathrm{Hb}$ levels and an increase in the prevalence of anaemia is not known with certainty. One possible mechanism might be that those with elevated CRP and/or AGP in a crosssectional survey may have, on average, either more frequent infections or infections of longer duration compared with those without elevated CRP and/or AGP. However, longitudinal data need to be examined to establish this. As seen in some other studies ${ }^{(12,13)}$, the results from the current survey show that the prevalence of anaemia varies depending on the presence or absence of inflammation. The overall estimated prevalence of anaemia in children 6-59.9 months of age was $48 \%$. If the prevalence of anaemia is restricted to children with normal CRP, the prevalence is estimated as $40 \%$; if restricted to children with normal AGP, 42\%; and if restricted to children with normal CRP and AGP, 39\%. Thus, there may be a need to account for inflammation in order to compare the prevalence of anaemia across populations or within a population over time.

While there are expert group recommendations on $\mathrm{Hb}$ cut-off values to define anaemia and adjustments based on trimester of pregnancy, altitude, cigarette smoking and ethnicity $^{(18)}$, there are no recommendations on how to account for inflammation in presenting anaemia results. Excluding survey participants with elevated markers of inflammation when presenting anaemia results can, however, be disadvantageous due to reduced sample sizes. Furthermore, excluding those with elevated APP may result in a biased sample because some population subgroups, most notably those of lower socio-economic status, may be differentially excluded due to their higher levels of inflammation and, in turn, those of lower socio-economic status tend to have a higher prevalence of anaemia.

Although the results of the present analysis correspond with findings reported in some studies ${ }^{(12,13)}$, other studies did not find associations between inflammation and anaemia $^{(14,15)}$. Whether or not an association between inflammation and anaemia is found in a population may be dependent on a variety of things, such as the presence of factors that may be causes of anaemia and/or inflammation. The most common causes of anaemia in less developed countries are Fe deficiency, parasitic infections and haemoglobinopathies. One possible explanation for the finding of an association between inflammation and anaemia in our data may be a high prevalence of certain parasitic infections in PNG, such as repeated malaria infections. In areas of high malaria endemicity, the constant exposure to infection may produce both inflammation and low $\mathrm{Hb}$. Although malaria thick blood smears were prepared for the children in our survey, these data could not be used in the present analysis because the malaria readings were deemed unreliable due to multiple discrepancies in slide readings. In PNG, malaria endemicity varies according to geography and elevation, with perennial malaria transmission occurring in lowland areas and localized malaria epidemics occurring in the highlands ${ }^{(24)}$. Even in areas with highly endemic malaria where adults and older children may have acquired partial immunity to malaria, clinical episodes of malaria are expected to occur more frequently in children less than 5 years of age ${ }^{(25)}$. Thus, the association between inflammation and anaemia could have occurred if there was a high prevalence of malaria infection among the survey participants.

As with all cross-sectional surveys, the current results should be interpreted cautiously. The results presented in our analysis are prevalence estimates and therefore the exposure-outcome directionality must be inferred from prospective research studies ${ }^{(23)}$. There is the potential for selection bias due to non-response in terms of participation in the survey and failure to obtain adequate blood specimens on a small percentage, although in the survey the overall response was relatively high.

In addition, there is the potential for misclassification of individuals on demographic and laboratory results, especially for laboratory results of proteins such as AGP for which the cut-off thresholds for abnormal values vary between studies. In the current analysis we used a cut-off value of $1.2 \mathrm{~g} / 1$ for AGP, as recommended by the laboratory that performed the measurements, resulting in a prevalence of elevated AGP of $32 \%$. Some researchers have used an AGP cut-off value of $1.0 \mathrm{~g} / \mathrm{l}^{(7)}$. If we apply this cut-off value to the data, we would find the prevalence of elevated AGP among the survey participants to be $56 \%$. Generally, if misclassification is non-differential, this would lead to a bias towards the null ${ }^{(23)}$. In the case of the AGP-anaemia association, using an AGP cut-off of $1.2 \mathrm{~g} / 1 v \cdot 1 \cdot 0 \mathrm{~g} / 1 \mathrm{did}$ not result in a marked difference in the prevalence odds ratio. The $1 \cdot 2 \mathrm{~g} / 1$ AGP cut-off resulted in a prevalence odds ratio of $2 \cdot 2$ (95\% CI $1 \cdot 6$, 3.0) for the AGP-anaemia association, whereas the $1.0 \mathrm{~g} / 1 \mathrm{AGP}$ cut-off results in a prevalence odds ratio of $2 \cdot 1(95 \%$ CI $1 \cdot 6,2 \cdot 9)$.

A further limitation is that the measure of association presented is the prevalence odds ratio, which will be an overestimate of the prevalence ratio given the high prevalence of anaemia in this population. For example, 
the crude prevalence odds ratio for the CRP-anaemia association presented in Table 1 is $3 \cdot 0$; the crude prevalence ratio for the CRP-anaemia association is 1.7 .

There is a need for more research on the association between inflammation and anaemia. Until further guidance is provided by expert groups, the authors recommend that cross-sectional surveys that collect information on anaemia and markers of inflammation present the prevalence of anaemia among those both with and without inflammation, in addition to the overall prevalence of anaemia and the prevalence of inflammation, in order to allow for comparisons between populations.

\section{Acknowledgements}

The funding sources for this survey were UNICEF and CDC. The findings and conclusions in this publication are those of the authors and do not necessarily represent the official position of the CDC or of the other organizations involved. The authors declare no conflicts of interest. All authors made a substantial contribution to the concept and design, acquisition or analysis and interpretation of data, were involved in drafting or revising the article for intellectual content, and have given approval to the final version of the article. The authors would like to thank the Department of Health Papua New Guinea, UNICEF Papua New Guinea and the University of Papua New Guinea for their continued support in conducting the first Papua New Guinea National Micronutrient Survey. The dedicated efforts of the field workers and data entry team are much appreciated. Special thanks are extended to Wila Saweri and Enoch Posani from the Department of Health and Clementine Yaman, Florence Addo and Karen Codling from UNICEF for their leadership in implementing the survey. The authors also thank Drs David Thurnham and Christine Northrop-Clewes for their insights into this issue of biomarkers of inflammation.

\section{References}

1. McLean E, Cogswell M, Egli I et al. (2009) Worldwide prevalence of anaemia, WHO Vitamin and Mineral Nutrition Information System, 1993-2005. Public Health Nutr 12, 444-454.

2. World Health Organization (2004) Focusing on Anaemia: Towards an Integrated Approach for Effective Anaemia Control. Joint Statement by the World Health Organization and the United National Children's Fund. Geneva: WHO.

3. Tolentino K \& Friedman JF (2007) An update on anemia in less developed countries. Am J Trop Med Hyg 77, 44-51.

4. Gabay C \& Kushner I (1999) Acute-phase proteins and other systemic responses to inflammation [erratum appears in N Engl J Med 1999 Apr 29;340(17):1376]. N Engl J Med 340, 448-454.

5. Pepys MB \& Hirschfield GM (2003) C-reactive protein: a critical update. J Clin Invest 111, 1805-1812.

6. Schultz DR \& Arnold PI (1990) Properties of four acute phase proteins: C-reactive protein, serum amyloid A protein,
1 -acid glycoprotein, and fibrinogen. Semin Arthritis Rheum 20, 129-147.

7. Thurnham DI, McCabe GP, Northrop-Clewes CA et al. (2003) Effects of subclinical infection on plasma retinol concentrations and assessment of prevalence of vitamin A deficiency: meta-analysis. Lancet 362, 2052-2058.

8. Hassan K, Sullivan KM, Yip R et al. (1997) Factors associated with anemia in refugee children. $J$ Nutr 127, 2194-2198.

9. Reeves JD, Yip R, Kiley VA et al. (1984) Iron deficiency in infants: the influence of mild antecedent infection. J Pediatr 105, 874-879.

10. Thurnham DI \& Northrop-Clewes CA (2007) Infection in the etiology of anemia. In Nutritional Anemia, pp. 231-256 [K Kraemer and MB Zimmermann, editors]. Basel: Sight \& Life Press.

11. Yip R \& Dallman PR (1988) The roles of inflammation and iron deficiency as causes of anemia. Am J Clin Nutr $\mathbf{4 8}$, $1295-1300$.

12. Maqsood M, Dancheck B, Gamble MV et al. (2004) Vitamin A deficiency and inflammatory markers among preschool children in the Republic of the Marshall Islands. Nutr J3, 21.

13. Rawat R, Stoltzfus RJ, Ntozini R et al. (2009) Influence of inflammation as measured by $\alpha-1$-acid glycoprotein on iron status indicators among HIV-positive postpartum Zimbabwean women. Eur J Clin Nutr 63, 787-793.

14. Wieringa FT, Dijkhuizen MA, West CE et al. (2002) Estimation of the effect of the acute phase response on indicators of micronutrient status in Indonesian infants. $J$ Nutr 132, 3061-3066.

15. Gamble MV, Palafox NA, Dancheck B et al. (2004) Relationship of vitamin A deficiency, iron deficiency, and inflammation to anemia among preschool children in the Republic of the Marshall Islands. Eur J Clin Nutr 58, 1396-1401.

16. Gorstein J, Sullivan K, Parvanta I et al. (2007) Indicators and Methods for Cross-Sectional Surveys of Vitamin and Mineral Status of Populations. Ottawa/Atlanta, GA: The Micronutrient Initiative/CDC.

17. World Health Organization (2001) Iron Deficiency Anaemia, Assessment, Prevention and Control: A Guide for Programme Managers. Geneva: WHO.

18. Sullivan KM, Mei Z, Grummer-Strawn L et al. (2008) Haemoglobin adjustments to define anaemia. Trop Med Int Health 13, 1267-1271.

19. Erhardt JG, Estes JE, Pfeiffer CM et al. (2004) Combined measurement of ferritin, soluble transferrin receptor, retinol binding protein, and C-reactive protein by an inexpensive, sensitive, and simple sandwich enzyme-linked immunosorbent assay technique. J Nutr 134, 3127-3132.

20. World Health Organization (2006) WHO Child Growth Standards: Length/Height-for-Age, Weight-for-Age, Weight-forLength, Weight-for-Height and Body Mass Index-for-Age: Methods and Development. Geneva: WHO.

21. Davis CE, Hyde JE, Bangdiwala SI et al. (1986) An example of dependencies among variables in a conditional logistic regression. In Modern Statistical Methods in Chronic Disease Epidemiology, pp. 140-147 [SH Moolgavkar and RL Prentice, editors]. New York: John Wiley \& Sons.

22. Kleinbaum DG (2002) Epidemiologic methods: the 'art' in the state of the art. J Clin Epidemiol 55, 1196-1200.

23. Kleinbaum DG, Barker N \& Sullivan KM (2005) ActivEpi Companion Textbook. New York: Springer.

24. Muller I, Bockarie M, Alpers M et al. (2003) The epidemiology of malaria in Papua New Guinea. Trends Parasitol 19, 253-259.

25. Imrie H, Fowkes FJI, Michon P et al. (2007) Low prevalence of an acute phase response in asymptomatic children from a malaria-endemic area of Papua New Guinea. Am J Trop Med Hyg 76, 280-284. 